\title{
Manipulation with Raw Ceramic Chimney Pipes
}

\begin{abstract}
Tomas Kellner ${ }^{1}$, Martin Kyncl ${ }^{1}$, Jiri Kyncl ${ }^{1}$, Michal Koptis ${ }^{1}$, Jan Urban ${ }^{1}$, Libor Beranek ${ }^{1}$, Miroslav Kotoucek ${ }^{2}$
${ }^{1}$ Faculty of Mechanical Engineering, Department of Machining, Process Planning and Metrology, CTU in Prague, Technicka 4, 16000 Praha 6, Czech Republic. E-mail: tomas.kellner@fs.cvut.cz, martin.kyncl@fs.cvut.cz, jiri.kyncl@fs.cvut.cz, michal.koptis@fs.cvut.cz, jan.urban@fs.cvut.cz, libor.beranek@fs.cvut.cz

${ }^{2}$ P-D Refractories CZ a.s., Nadrazni 218, 67963 Velke Opatovice, Czech Republic. E-mail: miroslav.kotoucek@pdgroup.com
\end{abstract}

\begin{abstract}
The aim of the paper is to describe the design of the manipulation system for the raw ceramic chimney pipe raw blank to avoid deformation of the pipe during handling and to increase the productivity of the manufacturing process. The process of manufacturing of a ceramic chimney pipe begins with extrusion of the raw refractory material. The extruded semi-finished product is then processed and then transferred by hand to a kiln car on which it is dried and fired. It is this manipulation that heavily contributes in the deformation of the fired chimney pipes. Due to the low stiffness of the raw ceramic chimney pipe, deformation occurs by manual handling. In order to avoid these deformations, it is necessary to design a suitable concept and construction solution of the manipulation system. Due to the nature of the chimney pipe, the automation of manipulation is rather difficult. The pipe is very soft in the raw state, its surface is rough, wet and greasy. This paper deals with the design of an optimal solution for the manipulation of ceramic chimney pipes, which will prevent the chimney pipe from deforming, negatively affecting its quality and making it possible to increase the productivity of ceramic chimney pipes production.
\end{abstract}

Keywords: Manipulation with Pipes, Refractory, Ceramic Chimney Pipe, Automation, Gripper Design

\section{Introduction}

Ceramic chimney pipes (CCP) are one of the main refractory products and are used as the main building part of domestic and industrial chimney systems. Compared to other materials, the ceramic pipes are characterized by high heat and corrosion resistance.

The ceramic chimney pipe is made of a refractory, water-insoluble mixture, most often based on silicates and clays. The basic elements of each ceramic composition are dense materials (for example, silica or aluminium oxides in crude or fired state) and a binder such as clay. Further, the composition may contain fillers and other substances which modify its properties in both raw and fired state. In the ceramic mixture, the most abundant is clay. Most ceramic products are then formed from the clay mixture by cold forming at different moisture levels of the crude mixture. For the production of some ceramic products, a liquid mixture is required, for others a mixture is almost dry. The drier mixture is mainly used for moulded ceramic products. After firing the raw product, the ceramic material itself is formed.

Ceramic chimney pipes are plastic after extrusion and are easily deformed. When the force is applied, deformation occurs on the CCP, which does not disappear when the force is applied. Long-term observations have shown that this deformation often occurs at the end of the production process, specifically after firing. If the force acting on the raw CCP is too high, the raw ceramic pipe is structurally broken and cracks are formed in the extruded mass of the blank. Due to the high pressure in the press (30-100 MPa), such cracks cannot be closed in any way. An inappropriate method of handling CCPs can result in high scrap rate of the final product, up to $10 \%$. However, the resulting scrap is actually even higher.
Chimney pipes with significant deformation, but below the given limit by customer, may be unusable for chimney system installation due to the nature, position and quantity of the deformations. If these significantly deformed pipes are included in the total scrap rate, the scrap rate will be up to $15 \%$. By optimizing the handling of raw $\mathrm{CCP}$, this number can be reduced by more than $50 \%$.

\section{Concept Design of Manipulation System}

The production of ceramic chimney pipes begins with the formation of a starting mixture of input raw material. This mixture is then brought to a horizontal or vertical press from which an endless blank is extruded. After the endless blank is pushed out by the press, it is necessary to cut this blank and then to process it. Subsequently, it is necessary to transport the finished ceramic chimney pipes in the raw state to the drying or kiln car. The problem lies in the way in which the ceramic chimney pipe is transferred to the car. With manual manipulation, the pipe is deformed in its central part, and after firing it exhibits significant shape deformities even on the end of pipe (due to stacking of the pipes on top of each other) that cannot be removed from the product.

There are several approaches to the design of end effectors (gripper). However, the easiest division is on two groups, the mechanical or vacuum grip of the pipe blank. The advantage of mechanical gripper is a more reliable grip, the disadvantage is the fact, there can be a greater force applying on differences in the shapes of the pipe blank and causing further deformations. The advantage of the vacuum gripping system is the minimization of the acting force, the great disadvantage is the instability of the grip with the possibility of loss of vacuum. After performing the risk analysis, the use of vacuum technology seems to be a more viable solution because of the minimal 
force impact on the raw CCP and the high variability of this solution. Another reason for the choice of vacuum manipulation is the fact that the chimney pipe blank as well as the final product is highly abrasive and any mechanical manipulator would need increased servicing. However, there is a need to design properly the entire system to ensure stable repeatability, despite CCP shape differences and their coarse, greasy and wet surfaces. This places a requirement on the design and selection of suitable effector elements. For the above reasons, it is necessary to replace the existing manual way of handling the new technology.

Regarding the manipulation device - manipulator, the conception can be divided for simplification into the main two groups. The first is the use of a single-purpose machine and the other is the use of a robotic arm. Therefore, it is a fixed or flexible automation. Due to the diversity of the dimensions of the chimney pipes, the combination of both types of automation was used. [6,8]

The robotic arm equipped with the gripper will manipulate the high turnover types of pipes. For the less frequent types of pipes, a manual manipulator would be used which would consist of a dedicated device and a manual manipulation device provided with a gripper. The expected benefits are reduced deformation and the ability to accelerate line tact when needed to increase production. If the chimney pipe blank had higher and stable mechanical properties, the design of a new manipulation technology would not pose a major challenge. However, due to the nature of the raw ceramic mass of the chimney pipe, handling without causing deformation and maintaining productivity is a difficult task.

\section{Testing of Suction Cup's Holding Force}

Before designing the constructional variants of the manipulation equipment, it was necessary to design and realize tests of the holding force (load capacity tests) of vacuum elements - suction cups. There are a number of suction cups for many applications, but they are mostly used on flat and clean (degreased) surfaces. There are suction cups specially designed for manipulation with preserved metal sheets, but the chimney pipes in addition are damp and greasy, rough and oval. Therefore, different suction cup shapes and sizes were selected for the tests.

Applying outer force on the ceramic chimney pipe by any load is not possible without distributing the applied force to the largest possible surface of the raw ceramic pipe - it would lead to creation of a crack in the CCP plastic extruded material. In Fig. 1, the scheme of radial and axial load tests can be seen. This test was designed to determine the maximum load capacity of the testing gripper (fixture) and vacuum elements in the radial and axial directions. This test also included verification that the suction cups do not damage the chimney pipe surface during handling, because, from the quality point of view, visible markings of the suction cup's edges are not acceptable. The test was carried out using a hydraulic jack and a load cell. Since it is a very soft material where the applied force has to be distributed to the largest possible area of the loaded chimney pipe. Therefore, pipe with a similar external diameter as the inner diameter of the CCP was used as a support element at radial load. In the axial direction, a large diameter circular plate was used to distribute the applied force across the upper edge of the chimney pipe. The suction cups were connected to ejectors generating a vacuum of up to $-95 \mathrm{kPa}$. [6]

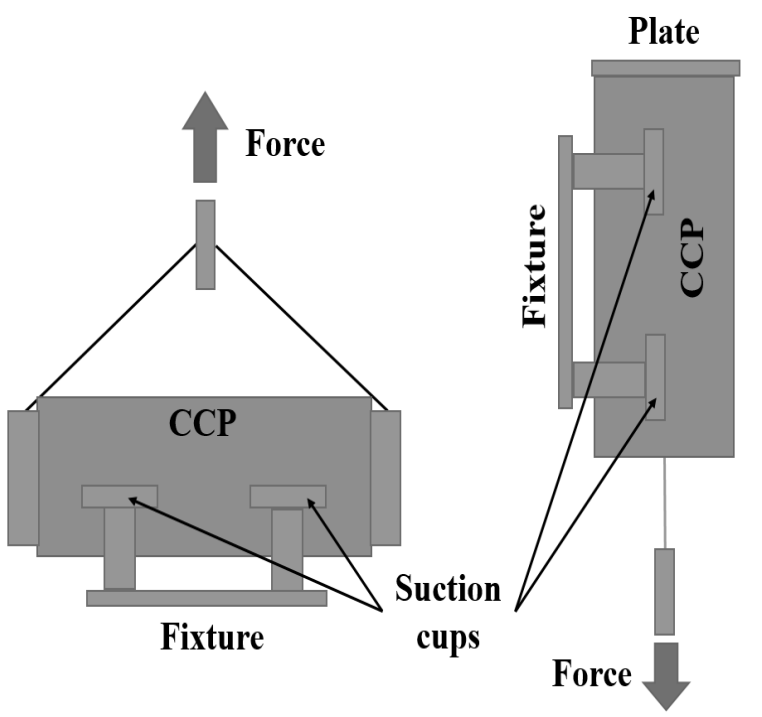

Fig. 1 Design of radial (left) and axial (right) load capacity tests

On the basis of the test results, oval suction cups designed for handling greasy metal sheets were chosen. Compared to sheet metal handling, however, it is necessary to choose a larger suction cup size. At the same time, however, the size of the suction cup must not be too large, in order to maintain the suction cup properties, to follow the round shape of the pipe. The performed test showed a smooth suction of selected vacuum suction cups with sufficient radial and axial load capacity. The test was conducted for different vacuum levels by controlling the compressed air leading to the ejector. At the compressed air source have been set 3, 4, 4.5 and 5 bars. For these values, the maximum load rating values on the test fixture were evaluated. However, higher vacuum values caused visible markings on the surface of the chimney pipe, which are not tolerable. Due to the state of the pipe's surface, the differences in holding force between lowest and highest feeding pressures were only about $20 \%$ of the measured force. Therefore, for the industrial application, it is better to have multiple suction cups with lower levels of vacuum to avoid generation of the markings. The best results were achieved by combination of feeding pressure of 2,5 - 3 bar (vacuum level approximately $-60 \mathrm{kPa}$ ) and four oval suction cups with dimension of $90 \times 35 \mathrm{~mm}$. With this setting, the holding force was high enough (above $400 \mathrm{~N}$ ) and also stable, even after conducting movement with the testing fixture. Thanks to the lower feeding pressure, there were almost non markings left on the surface of the pipe's blank. [7,10]

The results of the tests had been compared with theoretical calculations. The following calculations were made to establish the size of suction cup. Input variables: weight of chimney pipe $=\mathrm{m}=10 \mathrm{~kg}$, gravitational constant $=\mathrm{g}=9,81 \mathrm{~m} . \mathrm{s}^{2}$, Maximum achievable acceleration $=A_{t}=12 \mathrm{~m} \cdot \mathrm{s}^{2}$. Firstly, the axial force (1) and radial force 
(2) were calculated. After that, the Overall holding force $\left(F_{u}\right)$ was calculated (3) and from this equation, the force on one suction cup was calculated (4) with assumption of using 4 suction cups. From these results, the Minimum

required surface of suction cup could be calculated (5).

$$
\begin{aligned}
& F_{A X}=m \cdot\left(g+A_{t}\right)=10.21 .81=218.81[N](1) \\
& F_{R A D}=m \cdot A_{t}=10.12=120[N]
\end{aligned}
$$

Where:

$\mathrm{F}_{\mathrm{u}}$... Overall holding force $[\mathrm{N}]$

$\mathrm{k}$... Safety factor, $\mathrm{k}=1.5$

$\mathrm{f}_{\mathrm{k}}$... Grip coefficient, $\mathrm{f}_{\mathrm{k}}=0.6$

$$
F_{u 1}=\frac{F_{u}}{4}=\frac{628.21}{4}=157.1[N]
$$

Where:

$\mathrm{F}_{\mathrm{u} 1 \ldots \text {...Holding force per suction cup }[\mathrm{N}]}$

$$
A=\frac{F_{u 1}}{\Delta P}=\frac{157.1}{60000}=0.003135 \mathrm{~m}^{2} \doteq 31 \mathrm{~cm}^{2}
$$

Where:

A... The effective surface of the suction cup [cm] $\Delta \mathrm{P} \ldots$ Vacuum level $[\mathrm{Pa}]$

The calculated surface is around $31 \mathrm{~cm}^{2}$, which responds to rectangular suction cup with dimensions of 80 x $35 \mathrm{~mm}$. Through tests and also through calculation, it was verified, that 4 suction cups with dimensions at least $80 \times 35 \mathrm{~mm}$ will generate enough holding force to ensure grip through whole chimney pipe manipulation. This result corresponds with the results of the previously described tests. To further increase reliability of the grip throughout the manipulation process, oval suction cup with dimensions of $90 \times 35 \mathrm{~mm}$, originally designed for manipulation with preserved sheets, was chosen for design of final effectors (not the prototypes).

\section{Design of End Effectors}

Several designs of end effectors (grippers) have been proposed upon completion of the load capacity tests. Before the designing the solutions themselves, the necessary requirements for the whole system were defined. The most important requirements are the rigidity of the structure, low weight and the ability of the manipulation mechanism to handle the load without loss of grip during dynamic motions. [1]

For this reason, prototypes of grippers were designed in the first step (in parallel with the suction cups holding tests), which aimed to detect possible imperfections in the handling mechanism. After successful testing of functional variants (prototypes) it was possible to design the effectors for real operation. Due to the production of several types of ceramic chimney pipes, the effectors were also designed in various design solutions. The second step was the design of manually operated grippers to be serviced by employees. Thanks to the implementation of the vacuum balancer, there will be low physical effort needed from the employee. The third and final step was the design of the grippers for the robotic arm. [2]

The following subchapters will describe the individual design solutions:
- Prototype effectors - Effector with oval suction cups, steel plate effector, pocket effector

- Manual effectors - Effector for long CCP, effector for short CCP

- Robotic effectors - Effector for short CCP, effector for 4 short CCP and base ring

\subsection{Prototype Effector - Gripper with Oval Suction Cups}

In this variant of the prototype, it was a basic arrangement of suction cups on welded aluminium frame. The suction cups were placed at an angle of 80 degrees. The suction cups were chosen to be low profile with a size of $80 \times 40 \mathrm{~mm}$ with a total of four suction cups symmetrically positioned at the top of the chimney pipe in a horizontal position. The model of the effector prototype is shown in Fig. 2.

There were several shortcomings during the functional tests. Sufficient safety was considered when designing suction cups. But due to a character of the chimney pipe's surface, the tests with this prototype resulted in a low dynamic load bearing capacity. After switching on the vacuum supply, there was a rapid suction with high load capacity in static state. The problem occurred during a more rapid movement, where the nature of the surface with the design of the tested suction cup (low profile and too rigid) lead to loose of the grip. Another problem has been caused by the combination of relatively large low profile suction cups combined with a higher vacuum value. In addition to having a low dynamic load capacity after suction, the suction cups also showed clearly visible markings on the surface of the raw ceramic pipe. Surface quality is one of the guarded features of pipe quality, and therefore similar markings are unacceptable.
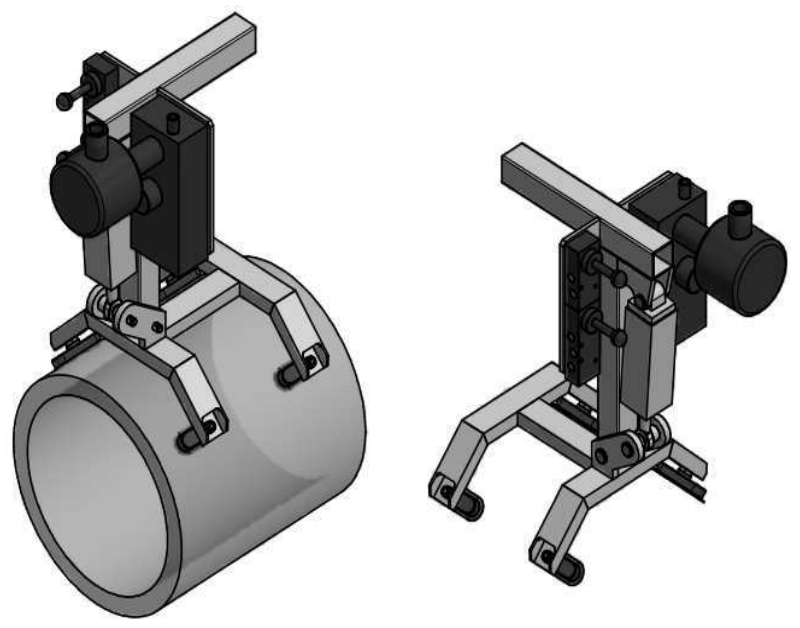

Fig. 2 Model of prototype effector with Oval Suction Cups 


\subsection{Prototype Effector - Steel Sheet Gripper}

The second version of the vacuum effector prototype was designed based on the results of the first version. First, the number and type of suction cups had changed. In this design, low-profile suction cups were used, this time round shape of $10 \mathrm{~mm}$ diameter with a total of 16 pieces. These suction cups were placed on a metal sheet that was shaped into an arc that follows the shape of the CCP.

During the first test, it has been found, that this type of suction cups were unsuitable for this application. If a product with a higher shape deformity is present on the manufacturing line, then it is not possible to pick the part with the proposed effector. The reason for this is the impossibility of any change in the position of the individual suction cups due to the positioning on the shaped sheet and also, the suction cups are too rigid to make up for the pipe's deformation. If there is an imperfect suction, even just one suction cup, the level of vacuum in the circuit is significantly compromised and the efficiency of the entire system will drop rapidly. In part, this problem could be solved by placing check valves on each of the suction cups or by using a multiple element vacuum source. Nevertheless, this solution will not solve the occurrence of the markings on the surface.

\subsection{Prototype Effector - Pocket Gripper}

Based on the existing knowledge, a third design of vacuum extraction has been proposed. Since the previous version was made of formed sheet metal whose shape copied the outer diameter of the CCP, it was decided that this sheet would be used to make this construction. The solution is based on the disassemble of the existing placement of individual suction cups, gluing of the sealing mass on the circumference of the whole sheet (including several strips in the middle part) and subsequent use of already produced holes for supplying vacuum to the working space. This results in a large-sized suction cup with a large work surface, as shown in Fig. 3.

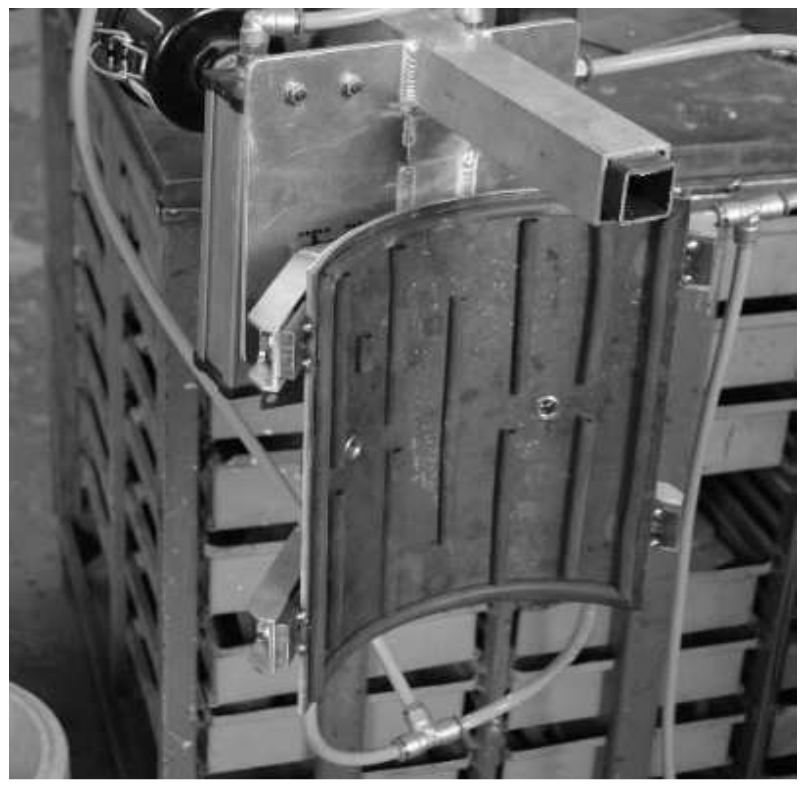

Fig. 3 Prototype of Pocket Gripper
The tests of this design itself were the most successful ones throughout testing of the prototypes. During the tests, there was always a smooth suction, excellent static load capacity and sufficient dynamic load capacity, which withstood even more severe shocks. The advantage of this solution is also the possibility of segmenting the vacuum into individual zones, by means of control valves, which prevent possible failure in a situation where an imperfect suction in a particular place would occur. However, the main disadvantage is the higher weight of the whole structure. Another problem has arisen after completing further dynamic tests and it is a lack of mechanical resistance of the sealant to abrasive wear caused by the raw chimney pipe material. Another disadvantage occurs, when pipe with already existing minimal deformations is manipulated. The shape of the pocket is given, so it cannot adapt to other then perfect shape of the pipe's blank. Despite very good holding capabilities, this design is not entirely usable for given task.

\subsection{Manual Effector - Gripper for Long CCP}

With the knowledge gathered through the results of the tests, the original design (effector with oval suction cups), made of aluminium profiles from Bosh Rexroth, was used. For this variant, both the design and the suction cups have been optimized (based on load capacity tests). This allows the effector to be converted to another product type (depending on size) if necessary. The construction was based on a basic supporting element, on which support arms are attached via adjustable joints. Suction cups are placed on these arms. The entire structure is reinforced with aluminium alloy sheet metal parts. Based on the results of the load-bearing tests, the entire structure was more reinforced with more robust bracing. The load capacity tests described in chapter 3 of this paper, were carried out in a static state, since a future fit for the robot arm is planned, a higher safety coefficient should be considered. Therefore, 8 suction cups are used in the modified design, due to expected heavier chimney pipes. The individual suction cups opposite each other are connected in two separated circuits. There are 4 suction cups in each circuit and one ejector as the vacuum source. Because of this type of connection, even if one suction cup will not be attached, that means the suction loss in all 4 suction cups, the other 4 suction cups will continue to produce enough grip to finish the manipulation movement. This fact was observed several times during testing of the gripper.

\subsection{Manual Effector - Gripper for Short CCP}

Compared to the previous effector, this effector is designed for short CCPs. Despite the shorter construction, the effector has same amount of suction cups. Because of this fact, it can be used for heavier variants of pipes. To ensure suction, it was necessary to maintain the number of suction cups. Again, the structure is made of aluminium profiles and aluminium and steel sheets. Each suction cup has its own arm with swivel joint and only opposed suction cups are connected. This makes it easy to adjust for CCP with slightly different diameters. The model of the effector is shown in Fig. . 


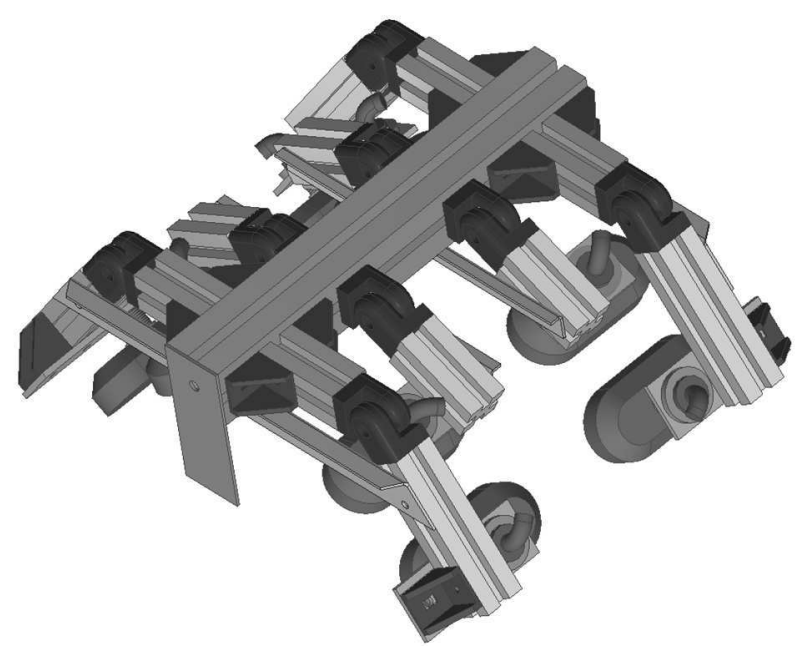

Fig. 4 Model of Manual Gripper for Short CCP

\subsection{Robotic Effector - Gripper for Short CCP}

Previous effectors are designed for manual manipulation of CCP types that are characterized by low production volumes. The effectors described in this chapter are designed for use in a automatic production line. For this reason, the emphasis is on the rigidity of the effectors while maintaining the concept of proven variant design. The structure is again realized with aluminium profiles, aluminium and steel sheets as bracings. Compared to manual effectors, there are more steel parts to increase stiffness. These designs of handling effectors include the parts needed for implementation with KUKA robots. The vacuum, in the case of robotic manipulation, is produced by a system of ejectors.
The first effector is used on the robotic arm to remove the CCP from the production line and place it on stacking platform in vertical position. After assembly of correct number of pipes ( 4 x CCP + base ring), this whole assembled unit will be moved on the kiln car using a second robot arm with a effector, as described in section 4.7. Unlike manual effector, not the joints, but the milled parts are used to further increase stiffness. This will result in greater rigidity. Reinforcements are from steel plates. [9]

The entire gripper is fixed with T-bolts to the machined steel flange. The flange is attached to a spacing tube that is threaded and has a pin hole on both sides. This tube is then attached to the robot flange.

\subsection{Robotic Effector - Gripper for 4 short CCP and Base Ring}

The design of this effector comes directly from the previous variant (chapter 4.6). The same segments are used to hold individual CCPs. The weight of the entire effector and flanges will be around $27 \mathrm{~kg}$ (without fasteners and pneumatic accessories). The flange for attaching the effector body to the distance tube is larger than in the case of a gripper for one CCP, but is of the same construction. A shorter distance tube was also used to minimalize momentum. Fig. 5 shows the design of the effector. The difference between this and previous variant is the segment for removing the base ring. It consists of a weldment made of machined sheets. This weldment serves as a base to determine the position of 4 smaller suction cups used for gripping the smaller base ring. Depending on differences in ceramic chimney pipes production, the base ring is not obligatory to use in the production process.

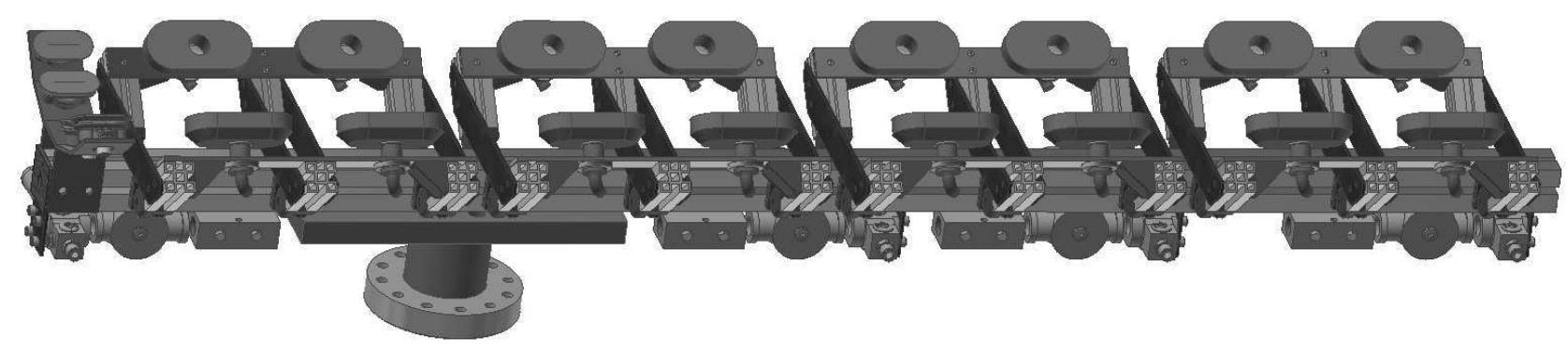

Fig. 5 Model of Robotic Gripper for 4 short CCP and Base Ring

\section{Design of Manipulation Device for Chimney Pipes Production}

In this paper, there was previously described, that two types of manipulation device are being used to handle the chimney pipes. The less common dimensions of CCP low volume production will be a single-purpose machine supplemented with a manual manipulator. Robotic arms incorporated into the automatic production line will be used for high turnover products.

The manipulation device for low volume production consists of the single-purpose device itself and a manual vacuum manipulator provided with the above-mentioned effectors. The manipulation of the CCP within this solution uses vacuum and suction cups, both on the device and on the manual manipulator. Again, vacuum is provided by series of ejectors. The handling process begins with the removal of the CCP blank from the conveyor (after extrusion and blank cutting) with a single-purpose device. Then, the CCP blank is moved at next station for cleaning of material residues after cutting. Another position of manipulation within suggested production line is the placement of the CCP blank in the device for creation of the sealing element - mostly referred to as tongue and groove. After the sealing element has been formed, the finished raw pipe is moved to the final position of the single-purpose machine where it is turned into a vertical position. Here, the handling process provided by the singlepurpose device ends and the handling process of the manual vacuum manipulator provided with the gripper begins. The employee removes the finished raw pipe from the end position with the manipulator and the vacuum balancer using and places it on the drying car. By creating this manual manipulator, employees are no longer subject 
to excessive physical stress. From a structural point of view, the main supporting part of the single-purpose device is a frame made of iron profiles and a movable axis with three grippers for CCP, whose horizontal position is unchangeable. This axis has two given positions, which is sufficient for the CCP to be manipulated to the appropriate positions. This makes controlling and function of this device's design very simple, easy to control, change configuration or maintain. When changing production to another type of pipe, only the grippers are replaced on the device and the technologic device creating the sealing element is also adjusted. Fig. 6 shows the single-purpose device model. [3]

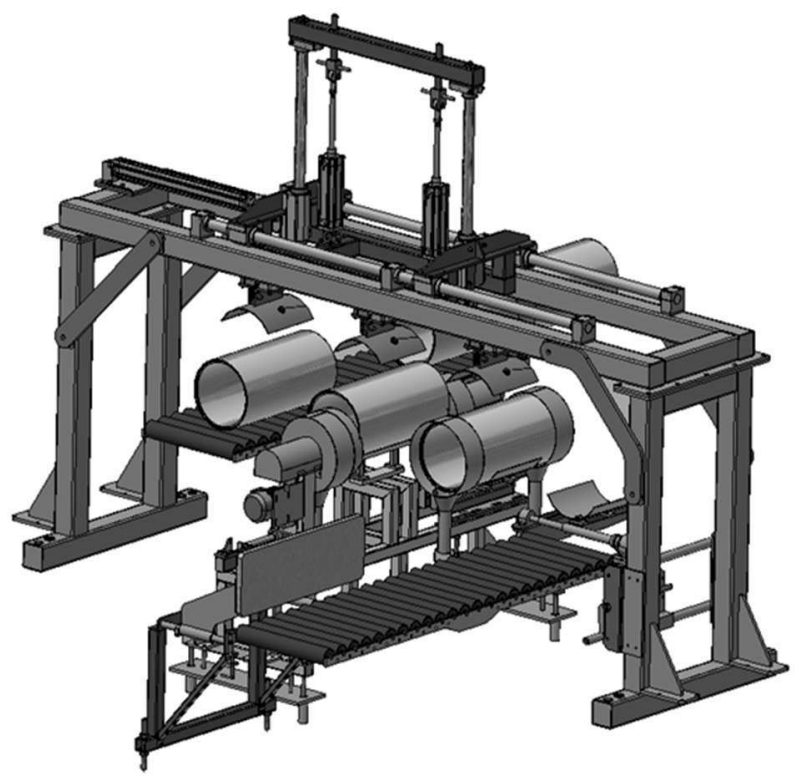

Fig. 6 Model of Manipulation device - Single-purpose device

A different manipulation system was chosen for high turnover production. After extrusion, the pipe extrusion is cut and moved to the first (smaller) robotic arm workspace by a conveyor. By the first robot, the pipe blank is picked from the conveyor by the gripper and manipulated to the stacking location. Then, the robot returns to the position to pick up the next pipe blank. As soon as 3 to 4 pipes (depending on the position on the kiln car and dimensions of the kiln) are placed at the stacking location (with or without base ring), the second robot comes forward. This second (larger) robot with a large gripper picks up all the pipes (and with base ring if present) at once and moves them to a predetermined target position on the surface of the kiln car. During this manipulation, the weight of the manipulated assembly and the maximum range of the robot needed to place the assembly in the furthest corner of the kiln car are the limiting factors of automation. Due to the very short tact times of the chimney pipes production line, which is usually in seconds, and the handling distances, it was necessary to use at least two industrial robots to achieve high productivity of the manufacturing process. Within this manipulation system design, great emphasis is placed on the ability of the gripper to achieve reliable grip with the CCP blank. Loss of vacuum would result in manipulated pipes falling during handling, and thus in stopping the production line. This risk was reduced within the presented design by the design of the gripper and the usage of proved vacuum accessories accompanied with tested wiring and assembly. $[3,4]$

\section{Conclusion}

This paper deals with design of manipulation with raw blank of ceramic chimney pipe. Unlike rigid products, the raw chimney pipe is plastic and easy to deform under any acting force. These deformations manifest themselves usually after the whole manufacturing process, after firing. That leads to increase of scrap rate and even inability to properly assemble chimney system from these manufactured ceramic chimney pipes. Therefore, the goal was to design technology of manipulation with raw ceramic chimney pipe blank. The blank itself isn't that easy to manipulate. In its state, it is wet, greasy, has very rough surface and the blanks usually has slightly different dimensions. After combining these facts, the pipe is very difficult to manipulate by classic fashion. The main goals of these paper were to design solutions of manipulation system for chimney pipes to minimize their deformation, mainly the design of grippers and manipulation systems.

The activities described in this article can be divided into 3 interconnected groups: design and testing of end effectors (grippers) for CCP manipulation, CCP load capacity tests and design of manipulation systems (device) with CCP.

Three prototype variants of grippers were designed. In the beginning of the research, the risk analysis took place to point out optimal mean of manipulation - mechanical or vacuum. Because of the nature of the raw chimney pipe, all the design are using vacuum devices for manipulation. These prototype effectors were used for static load carrying tests and the optimal design variant of the gripper effector was selected based on test results. At the same time as the prototypes, suction cups were tested for load capacity and a suitable type of suction cup was chosen based on these tests - a larger suction cup originally intended for handling oiled sheet metal. This resultant variant was subsequently modified and 4 grippers were designed for real industrial operation. Two grippers for manual operation and two effectors for robotic arm.

All four gripper variants were submitted to semi-operational test and the grippers met all the requirements established for handling the chimney pipes without undesirable deformation of the shape and surface of the pipe. These grippers will be used within the two proposed chimney pipe manipulation systems. For the less frequent dimensions of the pipes, a system using a single-purpose device together with a manually operated manipulator was designed. The synergy of these two manipulation technologies ensures sufficient productivity while maintaining the flexibility of the production line. For the more frequent dimensions of the chimney pipes, an automatic manipulation system using two robotic arms was designed to maximize the productivity of handling process and reduce the tact time of the production line. This places high demands on the quality of the manipulation system, especially on the grippers and the quality of suction vacuum elements. 


\section{Acknowledgement}

This work was supported by the governmental funding of Ministry of Industry and Trade, Czech Republic, program TRIO - project number FV10151.

\section{References}

[1] KYNCL, J., KELLNER, T., KUBIŠ, R. (2017). Tricanter production process optimization by digital factory simulation tools. In Manufacturing Technology. Vol. 17, No. 1, pp.49 - 53. ISSN 1213-2489.

[2] KYNCL, J. (2016). Digital Factory Simulation Tools. In Manufacturing Technology. Vol. 16, No. 2, pp.371 - 375. ISSN 1213-2489

[3] KOSTURIAK, J., GREGOR, M., MIČIETKA, B., MATUZSEK, J. (2000). Projektovanie výrobných systémov pre 21. storočí, Žilinská univerzita, 2000, pp. 397, ISBN 80-7100-553-3

[4] GÜNTHER, H.-O., TEMPELMEIER, H. (2006). Übungsbuch Produktion ung Logistik. Springer, 2006, pp. 248, ISBN 3-540-25704-7

[5] NEWMAN, S.T., ZHU, Z., DHOKIA, V.; SHOKRANI, A. (2015). Process planning for additive and subtractive manufacturing technologies. CIRP Annals - Manufacturing Technology 64 (2015), pp 467-470, ISSN 0007-8506
[6] VERMA, A., RAI, R. (2017). Sustainability-induced dual-level optimization of additive manufacturing process. The International Journal of Advanced Manufacturing Technology (2017), Vol. 88, Issue 5-8, pp 1945-1959, ISSN 02683768

[7] BEZERIANOS, A., BALAKRISHNAN, R. (2005). The vacuum. In: Proceedings of the SIGCHI conference on Human factors in computing systems. New York, New York, USA: ACM Press, 2005, 2005, s. 361. DOI: 10.1145/1054972.1055023. ISBN 1581139985

[8] SE-HAN, L., LASKY, A., VELINSKY, S. (2005). Mechanics and Robust Coordinated Control of the Automated Roadway Debris Vacuum Robotic Manipulator. Mechanics Based Design of Structures and Machines, vol. 33/no. 1, (2005), pp. 119145. ISSN: 1539-7734. DOI: 10.1081/SME200048324

[9] SHINTAKE, J., CACUCCIOLO, V., FLOREANO D., SHEA H. (2018). Soft Robotic Grippers. Advanced Materials. 2018, vol. 30/no. 29. DOI: 10.1002/adma.201707035. ISSN 09359648.

[10] MANTRIOTA, G. (2007). Theoretical model of the grasp with vacuum gripper. Mechanism and Machine Theory. 2007, 42(1), pp. 2-17. DOI: 10.1016/j.mechmachtheory.2006.03.003. ISSN: 0094114X. 